\title{
Je n'en peux plus de supporter toutes ces tâches administratives ! A moins que...
}

\author{
G. Meurette \\ (C) Lavoisier SAS 2016
}

« Je n'en peux plus de supporter toutes ces tâches administratives !»Cette plainte est probablement la plus prononcée par les médecins aujourd'hui. Elle s'accompagne généralement d'un cortège de superlatifs que la décence me retiendra de nommer dans le texte, mais qui traduit indiscutablement un agacement non dissimulé. On dépasse ici tous les clivages de spécialités et de modes d'exercices. S'il est un point sur lequel nous sommes tous d'accord, c'est bien cette dérive dans laquelle nous avons le sentiment frustrant de voir augmenter nos heures de tâches administratives au détriment de notre exercice médical. C'est dommage... Quelle performance de soin nous pourrions développer si demain (dans un monde idéal) on nous ôtait cette chape de plomb qui pèse sur nos épaules! Inutile de rêver, c'est l'inverse qui se produit. Vous le connaissez bien mes chers collègues : le fameux mille-feuilles qui doit bien atteindre les 2000 voire 3000 feuilles depuis qu'il se développe telle une épidémie dans notre quotidien.

Car il faut bien comprendre que gonfler nos tâches administratives génère aussi du boulot. Il faut ensuite programmer des réunions par dizaines, des « copil » (comprenez comités de pilotages) ; inventer des colonies d'acronymes pour des projets de développement, des procédures à écrire, des chiffres à interpréter, des nouvelles réunions à programmer. On vous présente des personnes qui dirigeront ces groupes dont vous n'osez pas demander de répéter la fonction exacte de peur de paraître idiot auprès de la majorité des membres du groupe... A l'inverse, ces gens semblent bien vous connaître, et vont vous expliquer qu'en changeant tel ou tel aspect de votre pratique, tout sera bien mieux. Chassez le naturel il revient au galop, je suis de nature optimiste. Pourtant, à chaque fois je pense (et ce sentiment est largement partagé avec mes collègues) que malgré des efforts réels pour suivre leur direction et défendre un point de vue médical, le dialogue peine à s'instaurer de façon constructive...

\footnotetext{
G. Meurette $(\bowtie)$

Unité de chirurgie pédiatrique, pôle FME, CHU Angers,

Institut des maladies de l'appareil digestif,

Hôtel-Dieu, 44000 Nantes

e-mail : gmeurette@hotmail.com
}

C'est sur ce sentiment mitigé que je débutais l'année 2016 (comme 2015 d'ailleurs); et puis parmi les articles scientifiques qui s'affichent en alerte sur mon bureau d'ordinateur ce matin apparaissait la lettre rédigée par Hirsch (directeur de l'assistance publique des hôpitaux de Paris) et al. [1] sur les attaques terroristes de Paris le 13 novembre dernier. Mon intérêt a été d'autant plus attiré que les échos venus jusqu'à mes oreilles de mes amis et connaissances qui avaient vécu cette terrible expérience au plus près était assez systématique : « Nous avons fait notre métier; ce pour quoi nous avons été formés ; de façon efficace et libérés de toute contrainte administrative (enfin) !».

Avant d'entreprendre ma lecture, j'y vois encore un argument confirmant l'idée que je me faisais de l'administration et ses dérives! Autant dire que je me lançais dans ma « LCA » (lecture critique d'article redoutée de nos jeunes étudiants) avec certains préjugés ; et pourtant je dois dire que cet article m'a invité à réviser mon point de vue. Il rapporte en effet le témoignage des attentats vécus par 4 corps de métiers : l'administration, le SAMU, les chirurgiens et les anesthésistes. L'éclairage des évènements par ces différents acteurs invite à plusieurs réflexions pour le lecteur extérieur que je représente.

D'abord, une impression indiscutable de professionnalisme de la part des acteurs médicaux (ce que je pensais fortement déjà). Paris concentre en effet le meilleur dans nombre de domaines médico-scientifiques. Alors pour l'urgence, les compétences médicales ne peuvent être qu'en pointe. Ce point a conforté ce que je savais déjà : pas de surprise!

Ensuite, un sentiment de fluidité et d'efficacité dans l'organisation des soins. On peut en juger par le nombre de patients pris en charge de façon très rapide (la prise en charge pré-hospitalière est ici très valorisée) mais par la capacité à délivrer les soins aux blessés admis dans les différents hôpitaux de façon « fluide ». Il ne s'agit pas ici d'agiter un drapeau tricolore ou de faire du chauvinisme, mais de souligner que fluidité et efficacité ne sont pas habituellement les qualificatifs qui caractérisent le mieux l'APHP dans la presse ou les actualités. Je fus sur ce point plutôt rassuré.

Enfin, en première lecture, une administration plutôt discrète comme je le pensais : tout s'est déroulé vite et la 
lenteur administrative n'était pas concernée par ce tourbillon soudain. Mais en seconde lecture, ma réflexion a été orientée par certaines phrases du texte qui suggèrent en filigrane que ce succès (c'en est un! on peut employer le terme) a pu aussi être possible par une politique d'organisation administrative certes contraignante pour tous, mais au final permettant que se déploie " naturellement » le dispositif médical avec l'efficacité qu'on lui reconnait aujourd'hui a posteriori. En d'autres termes, la vraie question à se poser est : " aurait-il été possible de faire aussi efficace si cette même administration n'avait pas préparé les ressources (humaines et matérielles) bien en amont pour finalement s'effacer assez logiquement le moment opportun ?».
L'objectif de cet édito n'était pas de répondre à cette question, mais de nous amener à la poser...

J'ai reposé sur mon bureau l'article que j'avais imprimé pour le lire attentivement et stabiloter (il faut bien avoir quelques vielles manies d'autres temps) ; j'ai consulté mon agenda (électronique cette fois) et décidé d'être à l'heure aux 2 réunions administratives prévues ce jour...

\section{Référence}

1. Hirsch M, Carli P, Nizard R, et al (2015) The medical response to multisite terrorist attacks in Paris. Lancet 385:2535-8 